\title{
G-PARKING FUNCTIONS AND TREE INVERSIONS
}

\author{
DAVID PERKINSON, QIAOYU YANG, AND KUAI YU
}

\begin{abstract}
A depth-first search version of Dhar's burning algorithm is used to give a bijection between the parking functions of a graph and labeled spanning trees, relating the degree of the parking function with the number of inversions of the spanning tree. Specializing to the complete graph solves a problem posed by R. Stanley.
\end{abstract}

\section{InTRODUCTION}

Let $G=(V, E)$ be a connected simple graph with vertex set $V=\{0, \ldots, n\}$ and edge set $E$. Fix a root vertex $r \in V$ and let SPT $(G)$ denote the set of spanning trees of $G$ rooted at $r$. We think of each element of $\operatorname{SPT}(G)$ as a directed graph in which all paths lead away from the root. If $i, j \in V$ and $i$ lies on the unique path from $r$ to $j$ in the rooted spanning tree $T$, then $i$ is an ancestor of $j$ and $j$ is a descendant of $i$ in $T$. If, in addition, there are no vertices between $i$ and $j$ on the path from the root, then $i$ is the parent of its child $j$, and $(i, j)$ is a directed edge of $T$.

Definition 1. An inversion of $T \in \operatorname{SPT}(G)$ is a pair of vertices $(i, j)$, such that $i$ is an ancestor of $j$ in $T$ and $i>j$. It is a $\kappa$-inversion if, in addition, $i$ is not the root and $i$ 's parent is adjacent to $j$ in $G$. The number of $\kappa$-inversions of $T$ is the tree's $\kappa$-number, denoted $\kappa(G, T)$.

Definition 2. A parking function for $G$ (with respect to the vertex $r$ ) is a function

$$
\mathcal{P}: V \backslash\{r\} \rightarrow \mathbb{N}
$$

such that for every nonempty set $S \subseteq V \backslash\{r\}$, there exists $i \in S$ such that $\mathcal{P}(i)<\operatorname{deg}_{S^{c}}(i)$, where $\operatorname{deg}_{S^{c}}(i)$ is the number of edges $\{i, j\}$ of $G$ with $j \notin S$ (including the possibility $j=r$ ). The degree of a parking function $\mathcal{P}$ is

$$
\operatorname{deg} \mathcal{P}:=\sum_{i \in V \backslash\{r\}} \mathcal{P}(i)
$$

The set of parking functions for $G$ is denoted by $\operatorname{PF}(G)$.

In this work, we introduce the DFS-burning algorithm. It is a melding of depth-first search with Dhar's burning algorithm [7] from the Abelian sandpile model, assigning a spanning tree to each parking function for $G$. Our main result is:

Theorem 3. The DFS-burning algorithm (Algorithm 1) gives a bijection $\phi: \operatorname{PF}(G) \rightarrow \operatorname{SPT}(G)$ such that

$$
\kappa(G, \phi(\mathcal{P}))=g-\operatorname{deg} \mathcal{P}
$$

where $g:=|E|-|V|+1$ is the circuit rank] of $G$. The inverse to $\phi$ is given by Algorithm 2 .

The reader is encouraged to refer to Figure 1 for an example.

\footnotetext{
${ }^{1}$ In the theory of parking functions, the circuit rank is often called the genus due to the role it plays in the Riemann-Roch theorem for graphs, [1].
} 
1.1. Background. Parking functions were originally defined in a study of hashing techniques in computer science, 14, phrased in terms of a problem involving preferences of drivers for parking spaces. Implicit in that definition is the restriction to complete graphs. Parking functions for general graphs have appeared in a variety of guises: in the Riemann-Roch theory for graphs, parking functions are known as reduced divisors, [1]; in the context of chip-firing games, they are known as superstable configurations, 12. Superstables are directly related to the recurrent configurations for Dhar's Abelian sandpile model on the graph, [12, Thm. 4.4], 7], and thus to the set of critical configurations in Biggs' dollar game, 4], and they serve as representatives for Lorenzini's group of components, [16, [17. The name "G-parking function" was introduced by Postnikov and Shapiro in 21. Parking functions in the case of a complete graph have appeared in the theory of symmetric functions, [11, 20], and hyperplane arrangements, [23] (for the latter, see [13] for an extension to more general graphs).

The Tutte polynomial for a simple graph $G$ is

$$
T(G, x, y)=\sum_{A \subseteq E}(x-1)^{c(A)-c(E)}(y-1)^{c(A)+|A|-|V|}
$$

where $c(A)$ is the number of connected components of the subgraph of $G$ with vertex set $V$ and edge set $A$. (For our purposes, we assume $G$ is connected, so $c(E)=1$.) Translating the work of Merino, 19, into the language of parking functions, $T(1, y)=\sum_{i=0}^{g} a_{i} y^{i}$ where $g-a_{i}$ is the number of parking functions for $G$ of degree $d$ and $g$ is the circuit rank of $G$. (Hence, $y^{g} T(1,1 / y)$ is the generating function for the parking functions by degree.) The definition of a $\kappa$-inversion is due to Gessel, [8], where it is introduced for the purpose of showing that $a_{i}$ is the number of spanning trees of $G$ with $\kappa$-number $i$. Theorem 3 may be regarded as an explanation of the coincidence.

Inversions and $\kappa$-inversions are the same when $G$ is a complete graph (or, more generally, if $G$ is a properly labeled threshold graph as explained in Section [3). In earlier work, Kreweras, 15, had already noticed that for a complete graph the number of trees with inversion number $a$ equals the number of parking functions of degree $g-a$. Stanley, [23, presents this result and poses the problem of finding a corresponding explicit bijection for the complete graph, $K_{n}$, that does not depend on recursing through bijections for $K_{i}$ for $i<n$, [23, Chapter 6, Exercise 4]. This problem was the motivation for our work and is generalized and solved by Theorem 3 . Note that although the algorithms of Theorem 3 use recursion, they recurse only through the vertices of a fixed graph. The runtime is $O(|V|+|E|)$, as it is for the usual depth-first search of a graph (and it would be a standard exercise to rewrite the algorithm using a stack and avoiding recursion without changing the runtime).

We were influenced by [2], which gives an exposition of the work of Cori and Le Borne in [6]. They describe an algorithm that gives a bijection between parking functions and spanning trees in which a parking function of degree $d$ is assigned a tree with external activity $g-d$. We are indebted to Farbod Shokrieh for explaining this work to us at the American Institute of Mathematics workshop on Generalizations of chip-firing and the critical group, July 2013. See $[3$ for a recursively defined permutation of labeled trees relating external activity to inversions. While preparing this manuscript we became aware of the work of Shin, [22, later subsumed in a paper by de Oliveira and Las Vergnas, 10, in which Stanley's problem had previously been solved. Roughly, depthfirst search is used to give a bijection between permutations and trees with no inversions. On the complete graph, permutations may be thought of as maximal-degree parking functions. To extend the bijection to arbitrary parking functions on the complete graph, a procedure is given for relabeling. The advantages of the bijection of Theorem 3 are (i) it applies to arbitrary (simple, 
connected, labeled) graphs, not only to complete graphs, and (ii) the algorithms providing the bijection and its inverse are less complicated, using little more than depth-first search, avoiding re-writing rules. We note that restricting our bijection to the case of complete graphs gives a substantially different bijection from that of [10] or [22].

Gessel and Sagan, 9], defines the neighbors-first search (NFS) of a graph and characterizes $\kappa$-inversions of a spanning tree in terms of edges that are externally active with respect to NFS, (Theorem 6.4, 9] ). The authors use NFS to describe a bijection between spanning trees and parking functions on complete graphs. The bijection relates the number of $\kappa$-inversions to a statistic on parking functions - thought of as hash functions - measuring the number of "probes" performed by a corresponding search protocol.

1.2. Organization. In Section 2 we describe and verify the algorithms providing the bijection, then prove Theorem 3. Section 3 considers threshold graphs, a class of graphs including the complete graphs. Proposition 10 in that section shows that for spanning trees of (suitably labeled) threshold graphs, every inversion is a $\kappa$-inversion.
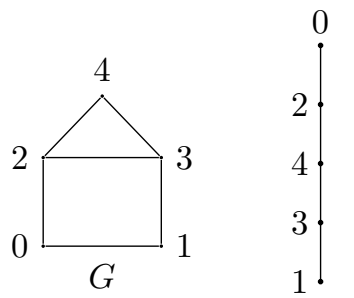

$$
\kappa=2
$$

$(0,0,0,0)$

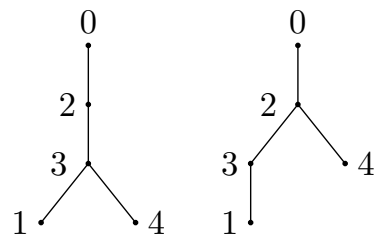

$(0,0,0,1)$

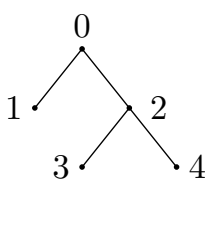

$\kappa=0$

$$
(1,0,1,0)
$$

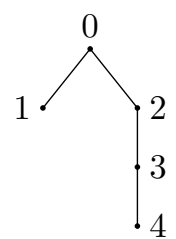

$$
\kappa=0
$$$$
(1,0,0,1)
$$
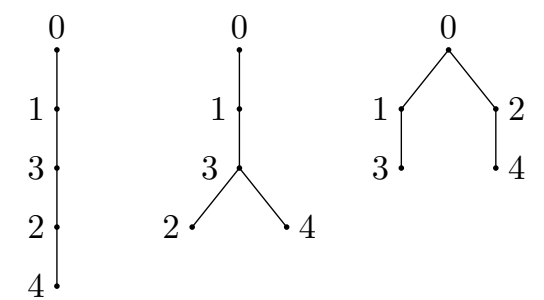

$\kappa=0$

$$
\kappa=0
$$

$(0,1,0,1)$

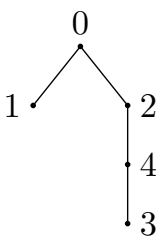

$$
\kappa=1
$$

Figure 1. The graph $G$ with its 11 spanning trees and their corresponding $\kappa$ numbers and parking functions (as provided by the DFS-burning algorithm). The root vertex is $r=0$. Each parking function $\mathcal{P}$ is written as vector with $i$-th component $\mathcal{P}(i)$.

Acknowledgements. The authors would like to thank Jim Fix and Farbod Shokrieh for helpful conversations. We thank Collin Perkinson for his comments. We also thank our anonymous referees for their thoughtful remarks. 


\section{Proof OF MAIN THEOREM}

In this section, it is assumed that $G$ is a connected simple graph with vertices $V=\{0, \ldots, n\}$ and fixed root vertex $r \in V$. We begin by describing the DFS-burning algorithm (where DFS stands for "depth-first search"). The idea is to imagine that a fire is started at the root vertex, $r$, of $G$ and spreads according to a depth-first rule, to be described below, along the edges until all vertices are burnt. A nonnegative function $\mathcal{P}$ is thought of as an allocation of drops of water to each non-root vertex 2. Suppose fire travels along an edge $e$ to a vertex $v$. If there are still drops of water on $v$, one drop will be used to "dampen" $e$, thus protecting $v$ from the flame, and the search backtracks. The depth-first rule is as follows: if there is no remaining water at $v$, then $e$ is marked, $v$ is burnt, and the fire proceeds from $v$ along an edge to the largest unburnt neighboring vertex. In the end, either (i) all vertices are burnt, $\mathcal{P}$ is a parking function, and the collection of marked edges forms a spanning tree, $T_{\mathcal{P}}$, (and the number of dampened edges is $\operatorname{deg} \mathcal{P}$ ) or (ii) the nonempty set of unburnt vertices, $S$, serves as a certificate that $\mathcal{P}$ is not a parking function: $\mathcal{P}(j) \geq \operatorname{deg}_{S^{c}}(j)$ for each $j \in S$. Algorithm 1 provides a precise statement of the DFS-algorithm. Its validity is established in Theorem 5

Example 4. Figure 2 illustrates a running of the DFS-burning algorithm. Vertex $i$ is labeled $v_{i}$. The value of the parking function $\mathcal{P}$ at $v_{i}$ is the $i$-th component of the vector $(0,0,1,0)$. The root vertex, $r=0=v_{0}$, is lit and fire spreads along the edge to the highest-numbered adjacent vertex, $v_{2}$. Since there are no drops of water on $v_{2}$, i.e., since $\mathcal{P}\left(v_{2}\right)=0$, the vertex $v_{2}$ is burnt and $\left(v_{0}, v_{2}\right)$ is added to the list of tree edges. Similarly, the fire spreads from $v_{2}$, causing $v_{4}$ to be burnt and $\left(v_{2}, v_{4}\right)$ to become a tree edge. The fire them attempts to spread to $v_{3}$, but the drop of water there is used to dampen the edge $\left(v_{4}, v_{3}\right)$. Backtracking to $v_{2}$, the fire then spreads to the remaining vertices. Note that the number of dampened edges is $\operatorname{deg} \mathcal{P}$.

The resulting spanning tree, $\phi(\mathcal{P})$, has inversions $\left(v_{2}, v_{1}\right)$ and $\left(v_{3}, v_{1}\right)$, but only $\left(v_{2}, v_{1}\right)$ is a $\kappa$-inversion since the parent of $v_{3}$ in the tree is $v_{2}$ and $\left\{v_{1}, v_{2}\right\}$ is not an edge in the graph. In accordance with Theorem 3, we have $g-\operatorname{deg} \mathcal{P}=2-1=1$.

Theorem 5 (DFS-bijection). After applying the DFS-burning algorithm to $\mathcal{P}: V \backslash\{r\} \rightarrow \mathbb{N}$, if burnt_vertices $=V$, then $\mathcal{P}$ is a parking function for $G$ and tree_edges forms a spanning tree of $G$. If burnt_vertices $\neq V$, the nonempty set $S:=V \backslash$ burnt_vertices has the property that $\mathcal{P}(j) \geq \operatorname{deg}_{S^{c}}(j)$ for all $j \in S$, certifying that $\mathcal{P}$ is not a parking function for $G$.

Associating to each parking function the spanning tree produced by the DFS-burning algorithm defines a bijection

The inverse is provided by Algorithm 2

$$
\phi: \operatorname{PF}(G) \rightarrow \operatorname{SPT}(G) .
$$

Proof. The DFS-burning algorithm must terminate since the function DFS_FROM() is called at most once per vertex and the loop starting at line 10 then eventually considers (perhaps after backtracking from a later call to DFS_FROM()) each adjacent vertex exactly once. Algorithm 2 terminates for similar reasons.

After applying the DFS-burning algorithm to $\mathcal{P}$, if every vertex is burnt, then a collection of $n$ edges is returned. These edges form a connected subgraph of $G$ containing all $n+1$ vertices and hence is a spanning tree. To see that in this case $\mathcal{P}$ is a parking function for $G$, suppose $S$ is a nonempty subset of $V \backslash\{r\}$. Suppose that $j$ is the first vertex of $S$ to be burnt. Just before $j$ is

${ }^{2}$ In [2], "firefighters" play the role of our drops of water. 


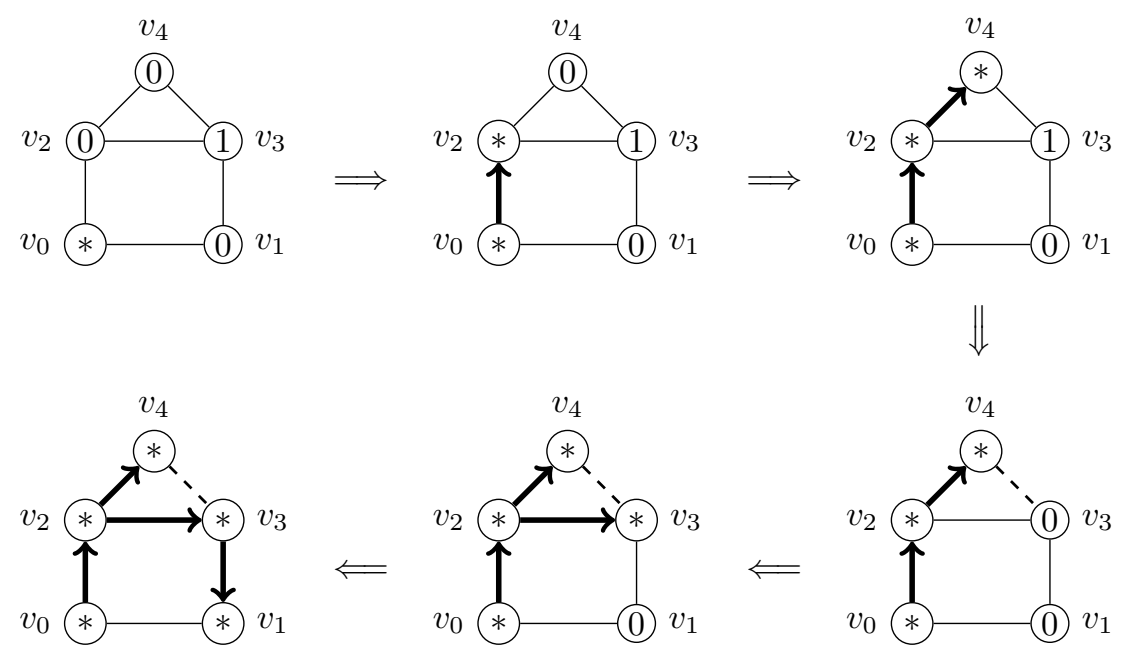

FiguRE 2. An application of the DFS-burning algorithm. Tree edges are arrows, dampened edges are dashed, and asterisks denote burnt vertices. To avoid confusion, vertex $i$ is labeled $v_{i}$. The root vertex is $r=v_{0}$. The values of the parking function are indicated on each vertex.

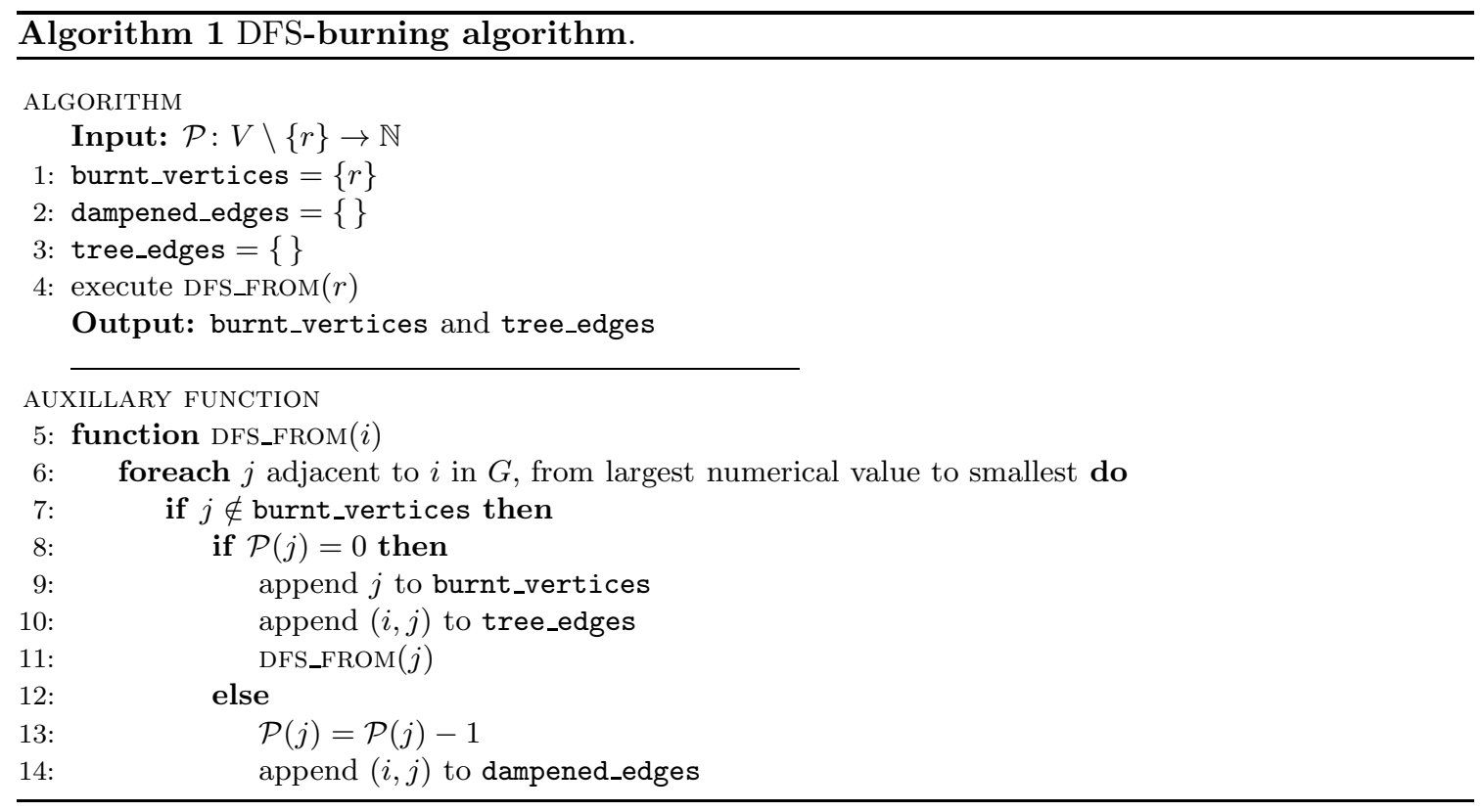

added to the list of burnt vertices (line 13), $\mathcal{P}(j)$ edges incident on $j$ will have been already added to the list of dampened edges. Then, just after $j$ is burnt, a new edge incident on $j$ is added to the list of tree edges. Each of these edges has the form $(i, j)$ where $i$ is a vertex burnt prior to $j$, and hence $i \notin S$. This shows that $\mathcal{P}(j)<\operatorname{deg}_{S^{c}}(j)$, as required. 


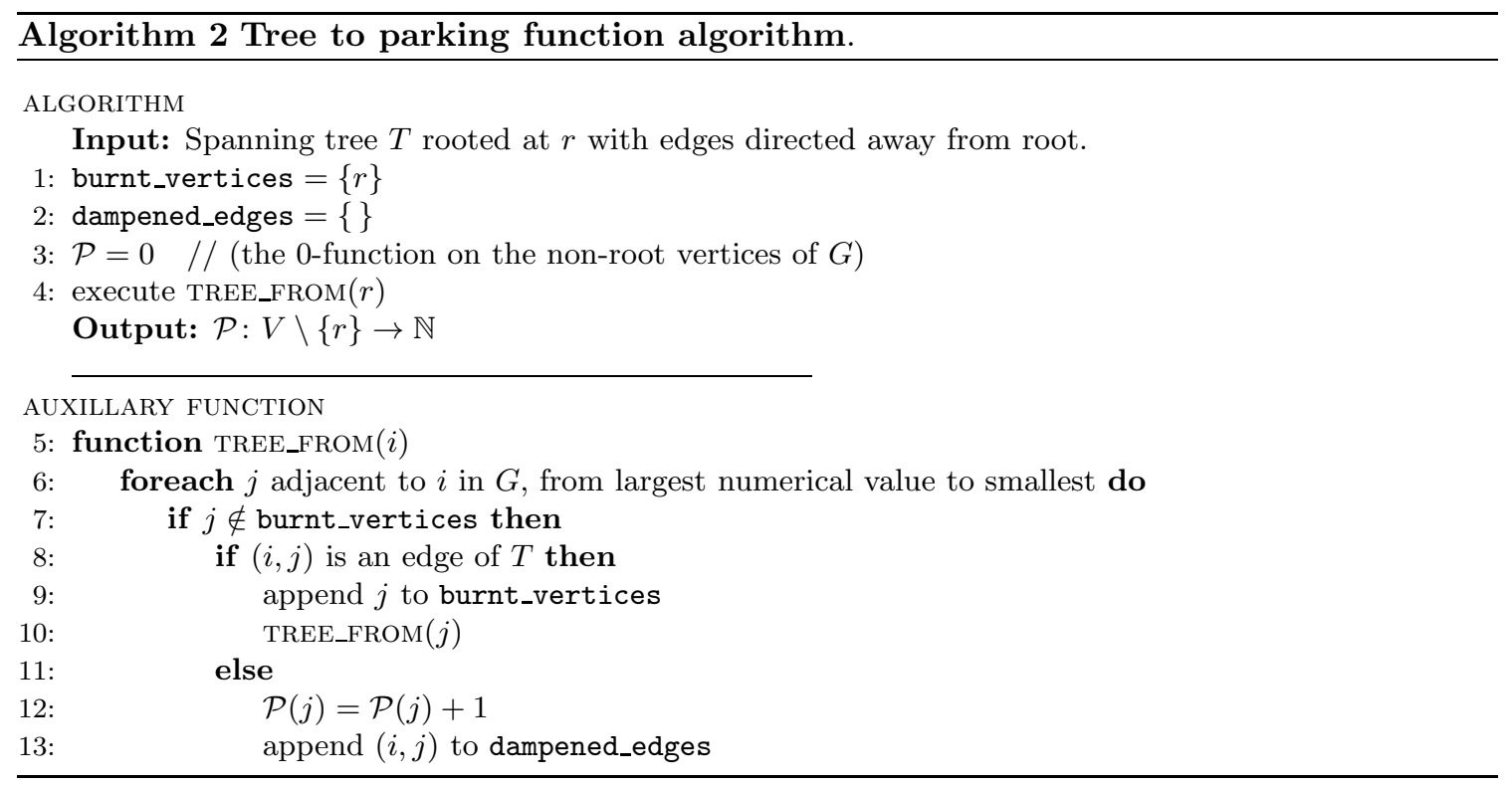

Now suppose that when the algorithm terminates, not every vertex is burnt. Let $S$ be the nonempty set of unburnt vertices. Take $j \in S$, and consider the set $I$ of vertices adjacent to $j$ but not in $S$. For each $i \in I$, the algorithm at some point added the edge $(i, j)$ to the set of dampened edges and decreased $\mathcal{P}(j)$ by one (while maintaining its nonnegativity). Thus, $\mathcal{P}(j) \geq \operatorname{deg}_{S^{c}}(j)$ (for the original input function $\mathcal{P}$ ), which shows that $\mathcal{P}$ is not a parking function for $G$.

We now show that the mapping $\phi$ is bijective with inverse provided by Algorithm 2 Consider the execution of the DFS-burning algorithm with input $\mathcal{P}$. When the for-loop at line 6 is entered, if $j$ is not a burnt vertex, an edge $e=(i, j)$ is added either to tree_edges or to dampened_edges. Make note of which case occurs, and in this way, create an ordered list $L(\mathcal{P})$ of directed edges, each edge marked as either a tree edge or dampened edge. The same edge may appear multiple times marked as dampened but appears at most once as a tree edge. Once an edge is marked as a tree edge, it will never appear later in the list with either marking. For each non-root vertex $j$, let $d(\mathcal{P}, j)$ denote the number of times $j$ appears as the head of a dampened edge in the list $L(\mathcal{P})$. Then $\mathcal{P}(j) \geq d(\mathcal{P}, j)$ for all $j$ and $\mathcal{P}(j)=d(\mathcal{P}, j)$ if $j$ appears as the head of a tree edge in $L(\mathcal{P})$. The function $\mathcal{P}$ is a parking function if and only if each non-root vertex appears as the head of tree edge in the list, in which case the edges marked as tree edges form a spanning tree and $\mathcal{P}(j)=d(\mathcal{P}, j)$ for each non-root vertex $j$.

For each $T \in \operatorname{SPT}(G)$ create a similar list $L(T)$ of marked edges using Algorithm 2 When a directed edge $e=(i, j)$ is considered in the for-loop, it is either part of $T$-in which case, mark $e$ as a tree edge - or it is added to dampened_edges, in which case, mark it as a dampened edge. In the end, each non-root vertex appears as the head of a tree edge exactly once, and that edge never appears subsequently in the list with either marking. Denote the output of the algorithm as $\mathcal{P}_{T}$. For each non-root vertex $j$, define $d(T, j)$ as above to be the number of times $j$ appears as the head of a dampened edge in $L(T)$, and note that $\mathcal{P}_{T}(j)=d(T, j)$.

For each $T \in \mathrm{SPT}(G)$, we claim $L\left(\mathcal{P}_{T}\right)=L(T)$. If not, consider the first entry at which the two lists differ. This entry must consist of the same edge, say $e=(i, j)$, but with different markings. 
First, suppose $e$ is marked as a tree edge in $L(T)$ and as a dampened edge in $L\left(\mathcal{P}_{T}\right)$. In that case, we get the contradiction:

$$
\mathcal{P}_{T}(j) \geq d\left(\mathcal{P}_{T}, j\right)>d(T, j)=\mathcal{P}_{T}(j) .
$$

Second, suppose $e$ is marked as a dampened edge in $L(T)$ and as a tree edge in $L\left(\mathcal{P}_{T}\right)$. In that case, we get the contradiction:

$$
\mathcal{P}_{T}(j)=d\left(\mathcal{P}_{T}, j\right)<d(T, j)=\mathcal{P}_{T}(j) .
$$

Since $L\left(\mathcal{P}_{T}\right)=L(T)$, it follows that $\phi\left(\mathcal{P}_{T}\right)=T$ and, thus, $\mathcal{P}_{T}$ is a parking function. In particular, the mapping $T \rightarrow \mathcal{P}_{T}$ is injective.

To see that $T \rightarrow \mathcal{P}_{T}$ is the inverse of $\phi$, it now suffices to show $\phi$ is injective. For each $\mathcal{P} \in \operatorname{PF}(G)$, we have seen that $\mathcal{P}$ and $\phi(\mathcal{P})$ are determined by $L(\mathcal{P})$. Suppose $\mathcal{P}, \mathcal{P}^{\prime} \in \operatorname{PF}(G)$ and $\mathcal{P} \neq \mathcal{P}^{\prime}$. It follows that $L(\mathcal{P}) \neq L\left(\mathcal{P}^{\prime}\right)$. Consider the first entry in which the lists differ. This entry is an edge $e=(i, j)$ marked as a tree edge in one list, say in $L(\mathcal{P})$, and a dampened edge in the other, $L\left(\mathcal{P}^{\prime}\right)$. If follows that $e$ appears in $\phi(\mathcal{P})$ but not in $\phi\left(\mathcal{P}^{\prime}\right)$. Thus, $\phi$ is injective.

We proceed to a proof of our main result, Theorem 3

Definition 6. The depth-first search tree (DFS-tree) of $G$, denoted $\operatorname{DFS}(G)$, is the output of the DFS-burning algorithm with input $\mathcal{P}=0$.

Lemma 7. Suppose $H$ is a connected graph obtained by deleting an edge of $\operatorname{DFS}(G)$ from $G$. Then the $\kappa$-inversions of $\operatorname{DFS}(H)$ as a subgraph of $G$ are the same as those as a subgraph of $H$ (assuming the same root for both $G$ and $H)$, and

$$
\kappa(G, \operatorname{DFS}(H))=\kappa(H, \operatorname{DFS}(H))=g-1,
$$

where $g=|E|-|V|+1=|E|-n$.

Proof. Let $T=\operatorname{DFS}(H)$. Trivially, $\kappa$-inversions of $T$ in $H$ are $\kappa$-inversions for $T$ in $G$. We prove the opposite inclusion by contradiction. Suppose $(i, j)$ is a $\kappa$-inversion for $T$ in $G$ but not in $H$. In other words, letting $i^{\prime}$ be the parent of $i$ in $T$, the edge $e=\left\{i^{\prime}, j\right\}$ is in $G$ but not in $H$, hence, $e$ is the edge of $\operatorname{DFS}(G)$ deleted to obtain $H$.

Since $G$ and $H$ differ only in the edge $e$, the depth-first searches of both $G$ and $H$ are the same up to the point at which the vertex $i^{\prime}$ of $e$ is reached. Next, since $i>j$, the depth-first search of $G$ travels from $i^{\prime}$ to $i$, i.e., $\left(i^{\prime}, i\right)$ is an edge of $\operatorname{DFS}(G)$. Subsequently, the path from $i$ to $j$ in $T$ must also be eventually added to $\operatorname{DFS}(G)$. Hence, $e$ cannot be in $\operatorname{DFS}(G)$, which is a contradiction.

To show that $\kappa(H, \operatorname{DFS}(H))=g-1$, suppose $e=\left\{i^{\prime}, j\right\}$ is an edge of $H$ but not an edge of $\operatorname{DFS}(H)$. Without loss of generality, assume $i^{\prime}$ is added to the list of burnt vertices before $j$ in the execution of the DFS-burning algorithm used to create $\operatorname{DFS}(H)$. Since $\left\{i^{\prime}, j\right\}$ is not in $\operatorname{DFS}(H)$, when the algorithm burns $j$, it has not yet backtracked to $i^{\prime}$. Hence, there is a path in $\operatorname{DFS}(H)$ from $i^{\prime}$ to $j$ of edges directed away from the root. If $i$ is the child of $i^{\prime}$ in this path, then $(i, j)$ is a $\kappa$-inversion for $\operatorname{DFS}(H)$. In this way, we get a bijection between edges of $H$ that are not edges of $\operatorname{DFS}(H)$ and $\kappa$-inversions of $\operatorname{DFS}(H)$. The result follows.

Proof of Theorem 3. Let $\mathcal{P} \in \operatorname{PF}(G)$. It remains to be shown that $\kappa(G, \phi(\mathcal{P}))=g-\operatorname{deg} \mathcal{P}$.

Let $D=\left\{e_{1}, \ldots, e_{k}\right\}$ be the dampened edges resulting from applying the DFS-burning algorithm to $\mathcal{P}$. We assume that these edges are listed in the order they were found by the algorithm, and note that $k=\operatorname{deg} \mathcal{P}$. Define $G_{0}:=G$, and for $\ell=1, \ldots, k$, let $G_{\ell}$ be the graph obtained from $G_{\ell-1}$ by removing edge $e_{\ell}$. Each $G_{i}$ contains $\phi(\mathcal{P})$ and is consequently connected. Further, for $G_{k}$, obtained from $G$ by removing all the dampened edges, we have $\operatorname{DFS}\left(G_{k}\right)=\phi(\mathcal{P})$. 
We now show that $e_{\ell} \in \operatorname{DFS}\left(G_{\ell-1}\right)$ for $0 \leq \ell<k$, from which the result follows by repeated application of Lemma 7. The key idea is that starting with any connected simple graph and any nonnegative function on the graph's non-root vertices, the first dampened edge created by the DFSburning algorithm is an edge in the graph's depth-first search tree. For example, $e_{1}$ is an edge of $\operatorname{DFS}(G)=\operatorname{DFS}\left(G_{0}\right)$. Define $\mathcal{P}_{0}=\mathcal{P}$, and for $1 \leq \ell<k$, define $\mathcal{P}_{\ell}: V \backslash\{r\} \rightarrow \mathbb{N}$ by

$$
\mathcal{P}_{\ell}(j)= \begin{cases}\mathcal{P}_{\ell-1}(j)-1 & \text { if } e_{\ell}=(i, j), \\ \mathcal{P}_{\ell-1}(j) & \text { otherwise. }\end{cases}
$$

Then $e_{\ell+1}$ is in $\operatorname{DFS}\left(G_{\ell}\right)$ since it is the first dampened edge when the algorithm is run with input $G_{\ell}$ and $\mathcal{P}_{\ell}$ (the full sequence of dampened edges being $e_{\ell+1}, \ldots, e_{k}$ ).

Corollary 8. $\kappa(G, \operatorname{DFS}(G))=g$.

Proof. Apply Theorem 3 to $\mathcal{P}=0$.

\section{THRESHOLD GRAPHS}

Threshold graphs are a family of graphs, including the complete graphs, introduced by Chvatal and Hammer [5]. For a comprehensive study, see [18. We show that if properly labeled, there is no distinction between inversions and $\kappa$-inversions for their spanning trees.

Definition 9. A graph is a threshold graph if it can be constructed from a graph with one vertex and no edges by repeatedly carrying out the following two steps:

- Add a dominating vertex: a vertex that is connected to every other existing vertex.

- Add an isolated vertex: a vertex that is not connected to any other existing vertex.

A threshold graph with more than one vertex is connected if and only if the last-added vertex is dominating. Each threshold graph is uniquely defined by its build sequence: a string starting with the symbol $*$ (for the initial vertex) followed by any string consisting of the letters $\mathbf{d}$ (for the addition of a dominating vertex) and $\mathbf{i}$ (for the addition of an isolated vertex). Thus, $*$ iddid describes the threshold graph pictured in Figure 3 formed from a single vertex by adding, in order, an isolated vertex, two dominating vertices, an isolated vertex, then a final dominating vertex. Omitting $*$ and reading left-to-right, group the consecutive sequences consisting entirely of a single

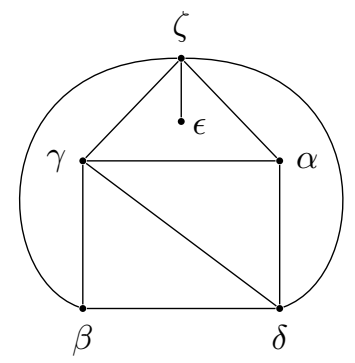

Figure 3 . The threshold graph with build sequence $*$ iddid. The vertices are labeled $\alpha, \beta, \gamma, \delta, \epsilon, \zeta$, in the order of the build sequence.

letter (either $\mathbf{d}$ or $\mathbf{i}$ ) into blocks, then include $*$ in the first block. Thus, the sequence of blocks for $* \mathbf{i d d i d}$ is $[* \mathbf{i}],[\mathbf{d d}],[\mathbf{i}],[\mathbf{d}]$. 
We say that a threshold graph is labeled by reverse degree sequence if its vertices are labeled by $0, \ldots, n$ in such a way that $\operatorname{deg}(i) \geq \operatorname{deg}(j)$ for each pair of vertices $i<j$. If there is more than one vertex with the same degree, the labeling is not unique. For example, for the graph in Figure 3 . (i) $\zeta$ must be labeled 0 , (ii) $\gamma, \delta$ must be labeled 1,2 in either order, (iii) $\alpha, \beta$ must be labeled 3,4 in either order, and (iv) $\epsilon$ must be labeled 5 .

Proposition 10. Let $G$ be a connected threshold graph labeled by reverse degree sequence, and let $T$ be a spanning tree of $G$. Then every inversion of $T$ is a $\kappa$-inversion.

Proof. First note that two vertices have the same degree in $G$ if and only if they belong to the same block of the build sequence for $G$. The degree of any vertex in a d-block is greater than the degree of any vertex in an i-block. Also, block-by-block, the degrees for vertices in successive $\mathbf{d}$-blocks increase from left-to-right, and the degrees for i-blocks decrease.

Let $(i, j)$ be an inversion of $T$, and let $i^{\prime}$ be the parent of $i$. We must show that $\left\{i^{\prime}, j\right\} \in E$, where $E$ denotes the set of edges of $G$. Since $i>j$ and $G$ is labeled by reverse degree sequence, $\operatorname{deg}(i) \leq \operatorname{deg}(j)$. If $\operatorname{deg}(i)=\operatorname{deg}(j)$, then $i$ and $j$ belong to the same block in the build sequence for $G$. In that case, not counting each other, $i$ and $j$ have the same neighbors. Hence, $\left\{i^{\prime}, j\right\} \in E$. Otherwise, $\operatorname{deg}(i)<\operatorname{deg}(j)$, and the result follows using the build sequence and considering cases:

- If $i$ and $j$ are both dominating, then $\operatorname{deg}(i)<\operatorname{deg}(j)$ implies $j$ follows $i$ in the build sequence, and thus $i^{\prime}$ must be adjacent to $j$.

- If $i$ is isolated and $j$ is dominating, then $i^{\prime}$ must be dominating. So $\left\{i^{\prime}, j\right\} \in E$ since every pair of dominating vertices are adjacent in $G$.

- If both $i$ and $j$ are isolated, then $j$ precedes $i$ in the build sequence and $i^{\prime}$ is a dominating vertex added after $i$. Hence, $\left\{i^{\prime}, j\right\} \in E$.

- It is not possible for $i$ to be dominating and $j$ to be isolated since $\operatorname{deg}(i)<\operatorname{deg}(j)$.

Remark 11. In light of Proposition 10, for a threshold graph one may replace $\kappa(G, \phi(\mathcal{P}))$ in Theorem 3 by the number of inversions of $\phi(\mathcal{P})$. Any labeling of a complete graph is by reverse degree sequence since the degree sequence in question is constant. Hence, Proposition 10 applies and shows that specializing Theorem 3 to the case of complete graphs gives a solution to a problem posed by Stanley [23, Chapter 6, Exercise 4]. 


\section{REFERENCES}

[1] M. Baker and S. Norine. Riemann-Roch and Abel-Jacobi theory on a finite graph. Adv. Math., 215(2):766-788, 2007.

[2] M. Baker and F. Shokrieh. Chip-firing games, potential theory on graphs, and spanning trees. J. Combin. Theory Ser. A, 120(1):164-182, 2013.

[3] J. S. Beissinger. On external activity and inversions in trees. J. Combin. Theory Ser. B, 33(1):87-92, 1982.

[4] N. L. Biggs. Chip-firing and the critical group of a graph. J. Algebraic Combin., 9(1):25-45, 1999.

[5] V. Chvátal and P. L. Hammer. Aggregation of inequalities in integer programming. In Studies in integer programming (Proc. Workshop, Bonn, 1975), pages 145-162. Ann. of Discrete Math., Vol. 1. North-Holland, Amsterdam, 1977.

[6] R. Cori and Y. Le Borgne. The sand-pile model and Tutte polynomials. Adv. in Appl. Math., 30(1-2):44-52, 2003. Formal power series and algebraic combinatorics (Scottsdale, AZ, 2001).

[7] D. Dhar. Theoretical studies of self-organized criticality. Phys. A, 369(1):29-70, 2006.

[8] I. M. Gessel. Enumerative applications of a decomposition for graphs and digraphs. Discrete Math., 139(13):257-271, 1995. Formal power series and algebraic combinatorics (Montreal, PQ, 1992).

[9] I. M. Gessel and B. E. Sagan. The Tutte polynomial of a graph, depth-first search, and simplicial complex partitions. Electron. J. Combin., 3(2):Research Paper 9, approx. 36 pp. 1996. The Foata Festschrift.

[10] A. Guedes de Oliveira and M. Las Vergnas. Parking functions and labeled trees. Sém. Lothar. Combin., 65:Art. B65e, 10, 2010/12.

[11] M. D. Haiman. Conjectures on the quotient ring by diagonal invariants. J. Algebraic Combin., 3(1):17-76, 1994.

[12] A. E. Holroyd, L. Levine, K. Mészáros, Y. Peres, J. Propp, and D. B. Wilson. Chip-firing and rotor-routing on directed graphs. In In and out of equilibrium. 2, volume 60 of Progr. Probab., pages 331-364. Birkhäuser, Basel, 2008.

[13] S. Hopkins and D. Perkinson. Bigraphical arrangements. To appear in Trans. Amer. Math. Soc.; eprint, arXiv:1212.4398, 2012.

[14] A. G. Konheim and B. Weiss. An occupancy discipline and applications. SIAM J. Applied Math., 14:1266-1274, 1966.

[15] G. Kreweras. Une famille de polynômes ayant plusieurs propriétés énumeratives. Period. Math. Hungar. 11(4):309-320, 1980.

[16] D. J. Lorenzini. Arithmetical graphs. Math. Ann., 285(3):481-501, 1989.

[17] D. J. Lorenzini. A finite group attached to the Laplacian of a graph. Discrete Math., 91(3):277-282, 1991.

[18] N. V. R. Mahadev and U. N. Peled. Threshold graphs and related topics, volume 56 of Annals of Discrete Mathematics. North-Holland Publishing Co., Amsterdam, 1995.

[19] C. Merino López. Chip firing and the Tutte polynomial. Ann. Comb., 1(3):253-259, 1997.

[20] J.-C. Novelli and J.-Y. Thibon. Hopf algebras and dendriform structures arising from parking functions. Fund. Math., 193(3):189-241, 2007.

[21] A. Postnikov and B. Shapiro. Trees, parking functions, syzygies, and deformations of monomial ideals. Trans. Amer. Math. Soc., 356(8):3109-3142 (electronic), 2004.

[22] H. Shin. A new bijection between forests and parking functions. eprint, arXiv:0810.0427, 2008.

[23] R. P. Stanley. An introduction to hyperplane arrangements. In Geometric combinatorics, volume 13 of IAS/Park City Math. Ser., pages 389-496. Amer. Math. Soc., Providence, RI, 2007.

E-mail address: davidp@reed.edu

E-mail address: yangq@reed.edu

E-mail address: kyu@reed.edu

Reed College, Portland OR, 97202 